\title{
Properties of Functions with Symmetric Points Involving Subordination
}

\author{
Malik Ali Raza (D), Syed Zakar Hussain Bukhari (D), Imtiaz Ahmed (D), Muhammad Ashfaq (D), \\ and Maryam Nazir iD
}

Department of Mathematics, Mirpur University of Science and Technology, Mirpur-10250(AJK), Pakistan

Correspondence should be addressed to Syed Zakar Hussain Bukhari; fatmi@must.edu.pk

Received 20 September 2021; Accepted 7 December 2021; Published 11 January 2022

Academic Editor: Wasim Ul-Haq

Copyright (C) 2022 Malik Ali Raza et al. This is an open access article distributed under the Creative Commons Attribution License, which permits unrestricted use, distribution, and reproduction in any medium, provided the original work is properly cited.

We study a new subclass of functions with symmetric points and derive an equivalent formulation of these functions in term of subordination. Moreover, we find coefficient estimates and discuss characterizations for functions belonging to this new class. We also obtain distortion and growth results. We relate our results with the existing literature of the subject.

\section{Introduction and Definitions}

Let $\mathscr{H}(\Delta)$ represent analytic functions $f$ in the disc $\Delta:=\{z$ $:|z|<1\}$ and $\mathscr{A} \subset \mathscr{H}(\Delta)$ be defined as:

$$
\mathscr{A}:=\left\{f \in \mathscr{H}(\Delta): f(z)=z+a_{2} z^{2}+\cdots(z \in \Delta)\right\} .
$$

Let $Q$ denote "Carathéodory functions" $h$ such that $h$ $(0)=1, \operatorname{Re}(h(z))>0$ and $h(z)=1+h_{1} z+h_{2} z^{2}+\cdots, z \in \Delta$. The Möbius function $k_{0}(z)=(1+z) /(1-z) \in \mathscr{Q}$ or its rotation acts as an extremal function for the class $Q$ and maps $\Delta$ onto $\operatorname{Re}\left(k_{0}(z)\right)>0$. Recall that $\mathbb{Q}(\varepsilon) \subset \mathbb{Q}, 0 \leq \varepsilon<1$ consists of functions $h \in \mathbb{Q}$ such that $\operatorname{Re}(h(z))>\varepsilon$ in $\Delta$. For $f, g \in \mathscr{H}$, we say that the function $f$ is subordinate to $g$ and write $f \prec g$, if for

$$
\Phi \in \mathscr{H}(\Delta) \text {, with } \Phi(0)=0 \text { and }|\Phi(z)|<1, f(z)=g(\Phi(z)) \text {. }
$$

For a univalent function $g, f<g$ if and only if $f(0)$ $=g(0)$ and $f(\Delta) \subset g(\Delta)$. For reference, see [1]. Applying subordination, Janowski [2] defined the class $\mathbb{Q}\left[A, A_{1}\right]$ for $-1 \leq A_{1}<A \leq 1$. A function $h \in \mathbb{Q}\left[A, A_{1}\right]$, if

$$
h(z)=\frac{1+A \Phi(z)}{1+A_{1} \Phi(z)} \prec \frac{1+A z}{1+A_{1} z}(z \in \Delta) .
$$

Geometrically, the image $h(\Delta)$ lies inside the disk centered on $\operatorname{Im}(z)=0$, and diameter ends at $h(-1)$ and $h(1)$. Clearly, $\mathbb{Q}\left[A, A_{1}\right] \subset \mathbb{Q}\left((1-A) /\left(1-A_{1}\right)\right)$. The class $\mathbb{Q}\left[A, A_{1}\right]$ is related with the class $\mathbb{Q}$ as: $h \in \mathbb{Q}$ iff, we write

$$
\frac{(A+1) h(z)-(A-1)}{\left(A_{1}+1\right) h(z)-\left(A_{1}-1\right)} \in \mathbb{Q}\left[A, A_{1}\right] .
$$

Also $\Lambda_{k}, \quad k \geq 0$ is given by $\Lambda_{k}=\{\Phi=u+i v: u>k$ $\left.\sqrt{(u-1)^{2}+v^{2}}\right\}$, represents various plane curves for the specific values of $k$. Let $\delta$ be the class of complex-valued injective functions and $\mathcal{S}^{*}$ represents the class of starlike functions whereas $\mathscr{C}$ denotes the class of convex functions. A function $f \in \mathcal{S}$ is close-to-convex, if and only if there exists a function $g \in \mathscr{C}$ such that

$$
\operatorname{Re}\left\{\frac{z f^{\prime}(z)}{g(z)}\right\} \in \mathbb{Q}, z \in \Delta
$$

We denote the class of close-to-convex functions by $\mathscr{K}$ . This class was introduced by Kaplan in [3]. Sakaguchi (see [4]) defined the class $\delta_{\mathrm{SP}}$ as: 
Definition 1. Let $f \in \mathcal{S}$. Then, $f \in \mathcal{S}_{S P}$, if

$$
\operatorname{Re}\left(\frac{2 z f^{\prime}(z)}{f(z)-f(-z)}\right)>0,(z \in \Delta) \text {. }
$$

For $f \in \mathscr{A}, f \in \mathscr{C}_{\mathrm{SP}}$ [5] iff $z f^{\prime} \in \mathcal{S}_{\mathrm{SP}}$, where $\mathscr{C}_{\mathrm{SP}}$ is the class of convex functions with respect to symmetric points. Various authors studied the class $\mathscr{C}_{\text {Sp }}$ and its subclasses, for detail, see $[3,6-8]$. Obviously, it represents the univalent functions. Moreover, it includes the class of convex and odd starlike functions, see [4]. This and other classes are investigated in the literature of the subject; for example, see [9-14].

Definition 2. Let $f$ be analytic in $\Delta$ defined by (1). We say that $f \in \mathscr{K}_{S P}(\varepsilon), 0 \leq \varepsilon<1$, if for $g \in \mathcal{S}^{*}(1 / 2)$ we have

$$
\operatorname{Re}\left(\frac{z^{2} f^{\prime}(z)}{g(z) g(-z)}\right)<-\varepsilon,(z \in \Delta) .
$$

For more details, see [15]. We see that $\mathscr{K}_{\mathrm{SP}}(0)=\mathscr{K}_{\mathrm{SP}}$, where $\mathscr{K}_{\mathrm{SP}}$ is the class of functions defined in [16]. We study a new class $\mathscr{K}_{\mathrm{SP}}(\varepsilon, \eta)$ involving $g \in S^{*}(1 / 2)$.

Definition 3. Let $f \in \mathscr{A}$. Then, $f$ $\in \mathscr{K}_{\mathrm{SP}}(\varepsilon, \eta), 0 \leq \varepsilon<1,0 \leq \eta \leq 1$ if for $g \in \mathcal{S}^{*}(1 / 2)$ we have

$$
\operatorname{Re}\left(\frac{z f^{\prime}(z)+\eta z^{2} f^{\prime \prime}(z)}{(1-\eta) G(z)+\eta z G^{\prime}(z)}\right)<-\varepsilon(z \in \Delta),
$$

where $G(z)=g(z) g(-z) / z$. By a simple calculations, we see that (8) is equivalent to

$$
\left|\frac{z f^{\prime}(z)+\eta z^{2} f^{\prime \prime}(z)}{(1-\eta) G(z)+\eta z G^{\prime}(z)}+1\right|<\left|\frac{z f^{\prime}(z)+\eta z^{2} f^{\prime \prime}(z)}{(1-\eta) G(z)+\eta z G^{\prime}(z)}-1+2 \varepsilon\right|,(z \in \Delta) .
$$

From [16], we have the following lemma.

Lemma 4. For $g \in \mathcal{S}^{*}(1 / 2)$ such that

$$
g(z)=z+\sum_{k=2}^{\infty} b_{k} z^{k},(z \in \Delta)
$$

if we put

$$
z G(z)=g(z) g(-z)=z^{2}+\sum_{k=1}^{\infty} c_{k+1} z^{2 k+1}
$$

where

$$
c_{k}=2 b_{2 k-1}-2 b_{2} b_{2 k-2}+. . \cdots+(-1)^{k} 2 b_{k-1} b_{k+1}+(-1)^{k+1} b_{k}^{2},
$$

then $G \in \mathcal{S}^{*}$.
Remark 5. Since $g \in \mathcal{S}^{*}(1 / 2)$, then Lemma 4 proves that $G$ $\epsilon \mathcal{S}^{*}$. Also from (8), we see that $\mathscr{K}_{\mathrm{SP}}(\varepsilon, \eta)$ contains closeto-convex functions.

\section{Main Results}

In the following theorem, we have an equivalent formulation of condition (9) in terms of subordination.

Theorem 6. A function $f \in \mathscr{K}_{S P}(\varepsilon, \eta)(0 \leq \varepsilon<1,0 \leq \eta \leq 1)$ iff for $g \in \mathcal{S}^{*}(1 / 2)$, we write

$$
\frac{-z f^{\prime}(z)-\eta z^{2} f^{\prime \prime}(z)}{(1-\eta) G(z)+\eta z G^{\prime}(z)}<\frac{1+(1-2 \varepsilon) z}{1-z},(z \in \Delta),
$$

where $G(z)=g(z) g(-z) / z$.

Proof. Let $f \in \mathscr{K}_{\mathrm{SP}}(\varepsilon, \eta)$. Then, for $g \in \mathcal{S}^{*}(1 / 2)$, we write

$$
\left|\frac{z f^{\prime}(z)+\eta z^{2} f^{\prime \prime}(z)}{(1-\eta) G(z)+\eta z G^{\prime}(z)}+1\right|<\left|\frac{z f^{\prime}(z)+\eta z^{2} f^{\prime \prime}(z)}{(1-\eta) G(z)+\eta z G^{\prime}(z)}-1+2 \varepsilon\right|,(z \in \Delta),
$$

or

$$
\operatorname{Re}\left(\frac{-z f^{\prime}(z)-\eta z^{2} f^{\prime \prime}(z)}{(1-\eta) G(z)+\eta z G^{\prime}(z)}\right)>\varepsilon,(z \in \Delta)
$$

where $G(z)=g(z) g(-z) / z$. Using subordination, we write

$$
H(z)=\frac{-z f^{\prime}(z)-\eta z^{2} f^{\prime \prime}(z)}{(1-\eta) G(z)+\eta z G^{\prime}(z)} \prec \frac{1+(1-2 \varepsilon) z}{1-z}, z \in \Delta,
$$

because $k_{0}(\varepsilon, z)=(1+(1-2 \varepsilon) z) /(1-z) \in \mathcal{S}$ and $H(0)=k_{0}$ $(\varepsilon, 0)=1$, where $G(z)=g(z) g(-z) / z$. Conversely, we assume that (13) holds. Then, there exists $\Phi$ with $\Phi(0)=0$ and $|\Phi(z)|<1$ such that

$$
\frac{-z f^{\prime}(z)-\eta z^{2} f^{\prime \prime}(z)}{(1-\eta) G(z)+\eta z G^{\prime}(z)}=\frac{1+(1-2 \varepsilon) \Phi(z)}{1-\Phi(z)} .
$$

Hence, using $|\Phi(z)|<1$, we obtain (9) equivalent to (8), so $f \in \mathscr{K}_{\mathrm{SP}}(\varepsilon, \eta)$.

Now, we prove sufficient conditions for $f \in \mathscr{K}_{\mathrm{SP}}(\varepsilon, \eta)$.

Theorem 7. Let $g \in \mathcal{S}^{*}(1 / 2)$ be a function given by (10) and $0 \leq \varepsilon<1,0 \leq \eta \leq 1$. If $f$ defined by (1) satisfies.

$$
2 \sum_{k=2}^{\infty}[k+\eta k(k-1)]\left|a_{k}\right|+(|1-2 \varepsilon|+1) \sum_{k=2}^{\infty}[2 \eta(k-1)+1]\left|c_{k}\right| \leq 2(1-\varepsilon),
$$

where the coefficients $c_{k}$ are given by (12), then $f \in \mathscr{K}_{S P}(\varepsilon, \eta)$. 
In particular, if

$$
\sum_{k=2}^{\infty}[k+\eta k(k-1)]\left|a_{k}\right| \leq 1-\varepsilon,
$$

then $f \in \mathscr{K}_{S P}(\varepsilon, \eta)$.

Proof. We set $F(\eta, z)=z f^{\prime}(z)+\eta z^{2} f^{\prime \prime}(z)$ for $f$ given by (1), $z G(z)=g(z) g(-z)$ where $g$ is defined by (10) and have

$$
\begin{aligned}
\Lambda= & \left|F(\eta, z)-(1-\eta) G(z)+\eta z G^{\prime}(z)\right| \\
& -\left|F(\eta, z)-(1-2 \eta)\left[(1-\eta) G(z)+\eta z G^{\prime}(z)\right]\right| \\
= & \left|\sum_{k=2}^{\infty}[k+\eta k(k-1)] a_{k} z^{k}-\sum_{k=2}^{\infty}[2 \eta(k-1)+1] c_{k} z^{2 k-1}\right| \\
& -\mid(2-2 \varepsilon) z+\sum_{k=2}^{\infty}[k+\eta k(k-1)] a_{k} z^{k} \\
& +(1-2 \varepsilon) \sum_{k=2}^{\infty}[2 \eta(k-1)+1] c_{k} z^{2 k-1} \mid .
\end{aligned}
$$

Hence, for $z \in \Delta$, we have the inequality

$$
\begin{aligned}
\Lambda \leq & \sum_{k=2}^{\infty}[k+\eta k(k-1)]\left|a_{k}\right||z|^{k}+\sum_{k=2}^{\infty}[2 \eta(k-1)+1]\left|c_{k}\right||z|^{2 k-1} \\
& -(2-2 \varepsilon)|z|+\sum_{k=2}^{\infty}[k+\eta k(k-1)]\left|a_{k}\right||z|^{k} \\
& +|1-2 \varepsilon| \sum_{k=2}^{\infty}[2 \eta(k-1)+1]\left|c_{k}\right||z|^{2 k-1} \\
= & -(2-2 \varepsilon)|z|+\sum_{k=2}^{\infty} 2[k+\eta k(k-1)]\left|a_{k}\right||z|^{k} \\
& +(|1-2 \varepsilon|+1) \sum_{k=2}^{\infty}[2 \eta(k-1)+1]\left|c_{k}\right||z|^{2 k-1} \\
\leq & -(2-2 \varepsilon)+\sum_{k=2}^{\infty} 2[k+\eta k(k-1)]\left|a_{k}\right|+(|1-2 \varepsilon|+1) \\
& \cdot \sum_{k=2}^{\infty}[2 \eta(k-1)+1]\left|c_{k}\right| \leq 0 .
\end{aligned}
$$

From these calculations, we see that $\Lambda<0$. Also by (20), we can write

$$
\begin{aligned}
& \left|F(\eta, z)-\left[(1-\eta) G(z)+\eta z G^{\prime}(z)\right]\right| \\
& <\left|F(\eta, z)+(1-2 \eta)\left[(1-\eta) G(z)+\eta z G^{\prime}(z)\right]\right|,
\end{aligned}
$$

which is equivalent to (9) and (8). Thus, $f \in \mathscr{K}_{\mathrm{SP}}(\varepsilon, \eta)$, and it completes the proof.
The next theorem deals with the coefficient estimates $f \epsilon$ $\mathscr{K}_{\mathrm{SP}}(\varepsilon, \eta)$.

Theorem 8. Let $0 \leq \varepsilon<1$ and $0 \leq \eta \leq 1$. Suppose that $f$ given by (1) and $g \in S^{*}(1 / 2)$ given by (10) are such that (8) holds. Then, for $k=2,3, \cdots$ we have

$$
\begin{aligned}
& 2 k^{2}\{1+\eta(2 k-1)\}^{2}-2(1-\varepsilon)^{2} \leq(1-\varepsilon) \\
& \cdot \sum_{k=2}^{n} 2(2 k-1) \eta_{k}^{2}\left|a_{2 k-1}\right|\left|c_{2 k-1}\right|+(|2 \varepsilon-1|+1) \eta_{k}^{2}\left|c_{2 k-1}\right|^{2}
\end{aligned}
$$

where $\eta_{k}=1+2 \eta(k-1)$ and $c_{k}$ is defined by (12). In particular, if $g(z)=z$, then

$$
k\left(1+\eta(2 k-1)\left|a_{2 k}\right| \leq 1-\varepsilon .\right.
$$

Proof. If $f \in \mathscr{K}_{\mathrm{SP}}(\varepsilon, \eta)$ for some $g \in S^{*}(1 / 2)$, then (9) holds. Using Lemma 4 , with $\alpha=\beta=1$, we have

$$
\frac{z f^{\prime}(z)+\eta z^{2} f^{\prime \prime}(z)}{(1-\eta) G(z)+\eta z G^{\prime}(z)}=\frac{1+(2 \varepsilon-1) z \Phi(z)}{1+z \Phi(z)}, z \in \Delta,
$$

where $\Phi$ is an analytic function in $\Delta,|\Phi(z)| \leq 1$ for $z \in \Delta$, and $G$ is given by (11). Then

$$
\begin{gathered}
{\left[\eta z^{2} f^{\prime \prime}(z)+z f^{\prime}(z)-(2 \varepsilon-1)\left\{(1-\eta) G(z)+\eta z G^{\prime}(z)\right\}\right]} \\
\cdot z \Phi(z)=(1-\eta) G(z)+\eta z G^{\prime}(z)-z f^{\prime}(z)-\eta z^{2} f^{\prime \prime}(z) .
\end{gathered}
$$

Now, $z \Phi(z)=\sum_{k=1}^{\infty} s^{k} z^{k}$. We see that $|\Phi(z)| \leq|z|$ for $z \epsilon$ $\Delta$. Thus

$$
\begin{gathered}
{\left[(2-2 \varepsilon) z+\sum_{k=2}^{\infty}[k+\eta k(k-1)] a_{k} z^{k}-(2 \varepsilon-1) \sum_{k=2}^{\infty}[2 \eta(k-1)+1] c_{k} z^{2 k-1}\right]} \\
\cdot \sum_{k=1}^{\infty} s^{k} z^{k}=\sum_{k=2}^{\infty}[2 \eta(k-1)+1] c_{k} z^{2 k-1}-\sum_{k=2}^{\infty}[k+\eta k(k-1)] a_{k} z^{k} .
\end{gathered}
$$

Equating coefficients in (27), for $k \geq 2$, we can also write

$$
\begin{aligned}
& {\left[(2-2 \varepsilon) z+\sum_{k=1}^{n-1} 2 k \eta_{2 k} a_{2 k} z^{2 k}+\sum_{k=2}^{n} \eta_{k}\left[(2 k-1) a_{2 k-1}-(2 \varepsilon-1) c_{2 k-1}\right] z^{2 k-1}\right]} \\
& \cdot z \Phi(z)=\sum_{k=2}^{n} \eta_{k}\left[c_{2 k-1}-(2 k-1) a_{2 k-1}\right] z^{2 k-1}-\sum_{k=1}^{k} 2 k \eta_{2 k} a_{2 k} z^{2 k} \\
& +\sum_{k=2 k+1}^{\infty} c_{k} z^{k},
\end{aligned}
$$

where $\eta_{k}=1+2 \eta(k-1)$ and $\eta_{2 k}=1+\eta(2 k-1)$. 
Then, we square and integrate along $|z|=r<1$. After using the fact $|\Phi(z)| \leq|z|<1$, we obtain

$$
\begin{aligned}
& \sum_{k=2}^{n}\left|\eta_{k}\left[c_{2 k-1}-(2 k-1) a_{2 k-1}\right]\right|^{2} r^{4 k-2} \\
& \quad+\sum_{k=1}^{k}\left|2 k \eta_{2 k} a_{2 k}\right|^{2} r^{4 k} \\
& \quad+\sum_{k=2 k+1}^{\infty}\left|c_{k}\right|^{2} r^{2 k}<|2-2 \varepsilon|^{2} r^{2}+\sum_{k=1}^{n-1}\left|2 k \eta_{2 k} a_{2 k}\right|^{2} r^{4 k} \\
& \quad+\sum_{k=2}^{n}\left|\eta_{k}\left[(2 k-1) a_{2 k-1}-(2 \varepsilon-1) c_{2 k-1}\right]\right|^{2} r^{4 k-2} .
\end{aligned}
$$

Letting $r \longrightarrow 1$, we have

$$
\begin{aligned}
& \sum_{k=2}^{n}\left|\eta_{k}\left[c_{2 k-1}-(2 k-1) a_{2 k-1}\right]\right|^{2}+\sum_{k=1}^{k}\left|2 k \eta_{2 k} a_{2 k}\right|^{2}<|2-2 \varepsilon|^{2} \\
& +\sum_{k=1}^{k-1}\left|2 k \eta_{2 k} a_{2 k}\right|^{2}+\sum_{k=2}^{n}\left|\eta_{k}\left[(2 k-1) a_{2 k-1}-(2 \varepsilon-1) c_{2 k-1}\right]\right|^{2} .
\end{aligned}
$$

Hence,

$$
\begin{aligned}
& 4 k^{2}\{1+\eta(2 k-1)\}^{2}-4(1-\varepsilon)^{2} \\
& \quad \leq \sum_{k=2}^{n}\left[\left|\eta_{k}\left[(2 k-1) a_{2 k-1}-(2 \varepsilon-1) c_{2 k-1}\right]\right|^{2}-\left|\eta_{k}\left[c_{2 k-1}-(2 k-1) a_{2 k-1}\right]\right|^{2}\right] \\
& \quad \leq 2(1-\varepsilon) \sum_{k=2}^{n} 2 \eta_{k}^{2}\left|a_{2 k-1} c_{2 k-1}\right|+(|2 \varepsilon-1|+1) \eta_{k}^{2}\left|c_{2 k-1}\right|^{2} .
\end{aligned}
$$

Thus, we have the inequality (23) which finishes the proof.

In the following theorem, we prove the growth and distortion theorems for $f$ in the class $\mathscr{K}_{\mathrm{SP}}(\varepsilon, \eta)$.

Theorem 9. If $f \in \mathscr{K}_{S P}(\varepsilon, \eta)$, where $0 \leq \varepsilon<1$ and $0 \leq \eta \leq 1$, then

$$
\chi_{1}(r, \varepsilon) \leq\left|f^{\prime}(z)\right| \leq \chi_{2}(r, \varepsilon)
$$

where

$$
\begin{gathered}
\chi_{1}(r, \varepsilon)=\frac{1-(1-2 \varepsilon) r}{(1+r)\left(1+r^{2}\right)}-\frac{(-1)^{1 / \eta}(1-\varepsilon)\{1+(1+r) \operatorname{arctanh} r}{r^{1 / \eta}(1+r)}, \\
\chi_{2}(r, \varepsilon)=\frac{1+(1-2 \varepsilon) r}{(1-r)\left(1-r^{2}\right)}-\frac{(-1)^{1 / \eta}(1-\varepsilon)}{4 r^{1 / \eta}} \\
\cdot \ln \left(\frac{1+r}{1-r}\right)-\frac{(1-\varepsilon)\left\{(-1)^{1 / \eta}(1-\varepsilon)+1\right\}}{2 r^{1 / \eta}(1-r)^{2}}
\end{gathered}
$$

Also, we have

$$
\chi_{3}(r, \varepsilon, \eta) \leq|f(z)| \leq \chi_{4}(r, \varepsilon, \eta)+\chi_{5}(r, \varepsilon, \eta),
$$

where

$$
\begin{aligned}
\left.\chi_{3}(r, \varepsilon, \eta)=1-\varepsilon\right) & \ln \frac{1+r}{\sqrt{1+r^{2}}} \\
+\varepsilon & \arctan r-\frac{\eta}{\eta-1} r^{-(1 / \eta)+1}-(-1)^{1 / \eta} \ln |1+r|, \\
\chi_{4}(r, \varepsilon, \eta)= & \frac{\varepsilon}{2} \ln \frac{1+r}{1-r}+\frac{(2-\varepsilon) r}{1-r}-\frac{\eta(-1)^{1 / \eta}(1-\varepsilon)}{4(\eta-1)} \\
\cdot & {\left[\ln \left(\frac{1+r}{1-r}\right) r^{-(1 / \eta)+1}-\ln \frac{|1+r|^{(-1)^{1 / \eta}}}{|1-r|}\right], } \\
\chi_{5}(r, \varepsilon, \eta)= & -\frac{1-\varepsilon}{2}\left[\frac{\eta}{\eta-1}\left[(-1)^{1 / \eta}+1\right] r^{-(1 / \eta)+1}\right. \\
& \left.-\left[(-1)^{1 / \eta}+1\right]\right] \ln |1-r|,
\end{aligned}
$$

and $|z|=r, 0 \leq r<1$

Proof. If $f \in \mathscr{K}_{\mathrm{SP}}(\varepsilon, \eta)$, then for $g \in S^{*}(1 / 2)$, (8) holds. It follows from Lemma 4 that $G$ in (11) is an odd starlike function. Then

$$
\frac{r}{1+r^{2}} \leq|G(z)| \leq \frac{r}{1-r^{2}},|z|=r, 0 \leq r<1
$$

$$
\frac{1-r^{2}}{\left(1+r^{2}\right)^{2}} \leq\left|G^{\prime}(z)\right| \leq \frac{1+r^{2}}{\left(1-r^{2}\right)^{2}},|z|=r, 0 \leq r<1 .
$$

For detail, see [17]. From (8), we obtain a function $h$ with real part greater then $\varepsilon$ such that

$$
\frac{z f^{\prime}(z)+\eta z^{2} f^{\prime \prime}(z)}{(1-\eta) G(z)+\eta z G^{\prime}(z)}=h(z), z \in \Delta .
$$

It is known, see [18], that

$$
\frac{1-(1-2 \varepsilon) r}{1+r} \leq|h(z)| \leq \frac{1+(1-2 \varepsilon) r}{1-r},|z|=r, 0 \leq r<1 .
$$

Thus, from (36), (38), and (39), we obtain (32). From (32) for $z=t e^{i \theta}$, we have 


$$
\begin{aligned}
&|f(z)|=\left|\int_{0}^{z} f^{\prime}(s) d s\right| \leq \int_{0}^{r}\left|f^{\prime}\left(t e^{i \theta}\right)\right| \\
& \cdot d t \leq \int_{0}^{r}\left[\frac{1+(1-2 \varepsilon) t}{(1-t)^{2}(1+t)}-\frac{(-1)^{1 / \eta}(1-\varepsilon)}{4 t^{1 / \eta}}\right. \\
&\left.\cdot \ln \left(\frac{1+r}{1-r}\right)-\frac{(1-\varepsilon)\left\{(-1)^{1 / \eta}(1-\varepsilon)+1\right\}}{2 t^{1 / \eta}(1-t)^{2}}\right] \\
& \cdot d t=\frac{\varepsilon}{2} \ln \frac{1+r}{1-r}+(2-\varepsilon) \frac{r}{1-r}-\frac{\eta(-1)^{1 / \eta}(1-\varepsilon)}{4(\eta-1)} \\
& \cdot\left[\ln \left(\frac{1+r}{1-r}\right) r^{-(1 / \eta)+1}-\ln \frac{|1+r|^{(-1)^{1 / \eta}}}{|1-r|}\right] \\
&-\frac{1-\varepsilon}{2}\left[\frac{\eta}{\eta-1}\left[(-1)^{1 / \eta}+1\right] r^{-(1 / \eta)+1}-\left[(-1)^{1 / \eta}+1\right]\right] \ln |1-r| .
\end{aligned}
$$

This gives us the right-hand side of the inequality (34). To prove the left-hand side of the inequality (34), we must show that it holds for the nearest point $f\left(z_{o}\right)$ from zero, where $\left|z_{o}\right|=r$ and $0<r<1$. Moreover, we have $|f(z)| \geq \mid$ $f\left(z_{o}\right) \mid$ for $|z|=r$. Since $f \in \mathscr{K}$, we know that the function $f$ is univalent in the unit disc $\Delta$. We conclude that the original image of the line segment

$$
\begin{aligned}
|f(z)|= & \int_{f(C)}|d \Phi|=\int_{C}\left|f^{\prime}(z)\right||d(z)| \\
\geq & \int_{0}^{r}\left[\frac{1-(1-2 \varepsilon) t}{(1+t)\left(1+t^{2}\right)}-\frac{(-1)^{1 / \eta}(1-\varepsilon)\{1+(1+r) \operatorname{arctanh} t}{t^{1 / \eta}(1+t)}\right] \\
& \cdot d t=(1-\varepsilon) \ln \frac{1+r}{\sqrt{1+r^{2}}} \\
& +\varepsilon \arctan r-\frac{\eta}{\eta-1} r^{-(1 / \eta)+1}-(-1)^{1 / \eta} \ln |1+r| .
\end{aligned}
$$

This finishes the proof of the inequality (34).

\section{Conclusions}

In this research, we studied a new subclass of functions with symmetric points and derived an equivalent formulation of these functions in terms of subordination. Moreover, we determined coefficient estimates and discussed characterizations for functions belonging to this new class. We also obtained distortion and growth results. We observed that our findings are related with the existing literature of the subject.

\section{Data Availability}

There is no data available.

\section{Disclosure}

The research is performed as part of the employment of the "Mirpur University of Science and Technology, Mirpur10250 (AJK), Pakistan.”

\section{Conflicts of Interest}

The authors declare that they have no conflicts of interest.

\section{References}

[1] S. S. Miller and P. T. Mocanu, Differential Subordinations: Theory and Applications, Marcel Dekker, Inc., New York, 2000.

[2] W. Janowski, "Some extremal problems for certain families of analytic functions I," Annales Polonici Mathematici, vol. 28, no. 3, pp. 297-326, 1973.

[3] T. N. Shanmugam, C. Ramachandram, and V. Ravichandran, "Fekete-Szegö problem for subclasses of starlike functions with respect to symmetric points," Bulletin of the Korean Mathematical Society, vol. 43, no. 3, pp. 589-598, 2006.

[4] K. Sakaguchi, "On a certain univalent mapping," Journal of the Mathematical Society of Japan, vol. 11, pp. 72-75, 1959.

[5] H. Silverman, "Integral means for univalent functions with negative coefficients," Houston Journal of Mathematics, vol. 23, no. 1, pp. 169-174, 1997.

[6] R. Parvatham and S. Radha, "On starlike and close-to-convex functions with respect to $k$-symmetric points," Indian Journal of Pure and Applied Mathematics, vol. 17, no. 9, pp. 1114-1122, 1986.

[7] V. Ravichandran, "Starlike and convex functions with respect to conjugate points," Acta Mathematica Academiae Paedagogicae Nyıregyháziensis, vol. 20, no. 1, pp. 31-37, 2004.

[8] Z.-G. Wang, "A new subclass of quasi-convex functions with respect to $k$-symmetric points," Lobachevskii Journal of Mathematics, vol. 19, pp. 41-50, 2005.

[9] S. Z. H. Bukhari, T. Bulboaca, and M. S. Shabbir, "Subordination and superordination results for analytic functions with respect to symmetrical points," Quaestiones Mathematicae, vol. 41, no. 1, pp. 65-79, 2018.

[10] S. Z. H. Bukhari, M. Nazir, and M. Raza, " $k$-symmetric points - some generalizations of the class of analytic functions with respect to k-symmetric points," Maejo International Journal of Science and Technology, vol. 1, no. 45, pp. 1-12, 2016.

[11] S. Z. H. Bukhari, M. Raza, and M. Nazir, " $k$-symmetric points - some generalizations of the class of analytic functions with respect to k-symmetric points," Hacettepe Journal of Mathematics and Statistics, vol. 1, no. 45, pp. 1$14,2016$.

[12] B. Seker and N. E. Cho, "A subclass of close-to-convex functions," Hacettepe Journal of Mathematics and Statistics, vol. 42, no. 4, pp. 373-379, 2013.

[13] H. M. Srivastava, S. Z. H. Bukhari, and M. Nazir, "A subclass of alpha-convex function with respect to $(2 j, k)$-symmetric conjugate points," Bulletin of the Iranian Mathematical Society, vol. 44, pp. 1227-1242, 2018.

[14] Z.-G. Wang and D.-Z. Chen, "On a subclass of close-to-convex functions," Hacettepe Journal of Mathematics and Statistics, vol. 38, no. 2, pp. 95-101, 2009.

[15] J. Kowalczyk and E. Les-Bomba, "On a subclass of close-toconvex functions," Applied Mathematics Letters, vol. 23, no. 10 , pp. $1147-1151,2010$. 
[16] C. Gao and S. Zhaou, "On a class of analytic functions related to the starlike functions," Kyungpook Mathematical Journal, vol. 45, pp. 123-130, 2005.

[17] P. L. Duren, Univalent Functions, Springer-Verlag, New York, 1983.

[18] A. W. Goodman, Univalent Functions, vol. 1, Mariner Publishing House, Tampa, Florida, 1983. 